\title{
Initial changes in alveolar bone volume for sham-operated and ovariectomized rats in ligature-induced experimental periodontitis
}

\author{
Jing Dai ${ }^{1} \cdot$ Yihui $\mathrm{Ma}^{1} \cdot$ Miusi Shi $^{1} \cdot$ Zhengguo Cao $^{1} \cdot$ Yufeng Zhang ${ }^{1,2}$. \\ Richard J. Miron ${ }^{3}$
}

Received: 18 November 2014 / Accepted: 3 July 2015 / Published online: 17 July 2015

(C) Springer-Verlag Berlin Heidelberg 2015

\begin{abstract}
Objectives Osteoporosis is a disease characterized by a reduction in bone mass, poor bone strength, and microarchitectural deterioration primarily in postmenopausal women. With respect to periodontal disease, osteoporosis is thought to contribute to pre-existing alveolar degeneration although the association between both diseases is not fully characterized. The aim of the present study was to observe the initial changes in mandibular alveolar bone for sham-operated and ovariectomized (OVX) rats in ligature-induced experimental periodontitis.

Materials and methods A total of 64 Wistar rats (7 weeks of age, 180-200 g) were used in this study (32 control shamoperated animals+ligature placement, 32 OVX animals + ligature placement). Following an 8-week period to induce an OVX model, micro-CT analysis was performed to calculate vertical and furcation bone loss of mandibular first molars at time points $0,3,7$, and 11 days following ligature placement (six animals per group per time point). Furthermore, histological analysis was performed to calculate the loss of alveolar bone crest height from the cemento-enamel junction, and
\end{abstract}

Yufeng Zhang

zyf@whu.edu.cn

1 The State Key Laboratory Breeding Base of Basic Science of Stomatology (Hubei-MOST) and Key Laboratory of Oral Biomedicine Ministry of Education, School and Hospital of Stomatology, Wuhan University, 237 Luoyu Road, Wuhan 430079, People's Republic of China

2 Department of Oral Implantology, School of Stomatology, Wuhan University, Wuhan 430079, China

3 Department of Periodontology, University of Bern, Bern, Switzerland tartrate-resistant acid phosphatase (TRAP) staining was utilized to calculate the number of osteoclasts.

Results The results from the present study demonstrate that OVX animals showed significant vertical bone loss at all time points when compared to control sham-operated animals. In the furcation area, no significant difference in bone loss was observed between sham-operated and OVX animals at 0,3 , and 7 days; however by 11 days, a significant decrease in bone volume/total volume and trabecular thickness was observed in the OVX group. The histological analysis also revealed that alveolar bone crest height was significantly reduced in OVX animals, and TRAP staining further revealed the greater number of multinucleated osteoclasts peaking at 3 days postligature placement.

Conclusion The results from the present study demonstrate a direct correlation between the osteoporotic phenotype and the progression of periodontal breakdown in a diseased-induced animal model.

Clinical relevance It may be suggested that an osteoporotic phenotype has the potential to speed periodontal breakdown and thus contributes to the overall degeneration of the periodontium in patients suffering from postmenopausal bone loss. Future research from human clinical studies are necessary to further understand the relationship between periodontal disease and osteoporosis.

Keywords Osteoporosis · Periodontal disease · Periodontitis · Ovariectomy

\section{Introduction}

Osteoporosis is a worldwide health care issue characterized by reduction in bone mass, poor bone strength, and microarchitectural deterioration in trabecular and cortical 
bone. The disease is known to progress with increasing age, and the primary cause is thought to be derived by an imbalance between bone-forming osteoblasts and bone-resorbing osteoclasts due primarily from postmenopausal estrogen deficiencies [1-5]. Over 200 million people are now estimated to be suffering from the disease worldwide with the great majority being white or Asian women over the age of 65 [6]. Commonly utilized pharmacological agents for the treatment of osteoporosis include anabolic agents capable of increasing bone formation such as parathyroid hormone and the use of anti-resorbing agents such as bisphosphonates, calcitonin, RANKL inhibitors, raloxifene, and estrogen replacement therapy [7]. Despite these many options, there exist no single option capable of fully restoring lost bone, and the use of bisphosphonate therapy (the leading treatment options for prevention of bone mass in osteoporotic patients) is associated with a number of side effects including halting the bone remodeling cycle.

Interestingly, growing clinical evidence has suggested that a relationship between osteoporosis and periodontal disease exist [8-14]. In one of the first well-documented studies investigating this relationship, it was found that osteoporosis had no significant effect with respect to plaque and gingival bleeding but was associated with a significantly greater loss of attachment in osteoporotic women [10]. Passos et al. compared different diagnostic criteria for periodontal disease that were used to study the association between periodontal disease and osteoporosis [8]. The results from their work emphasize that the frequency of periodontal disease may be influenced by different outcome measurements associated between osteoporosis and periodontal disease. More recently, Takahasi et al. studied the association between periodontitis and systemic bone mineral density in Japanese community-dwelling postmenopausal women [13]. They evaluated the association between loss of attachment and bone mineral density (BMD) of the lumbar vertebrae and femur in 374 postmenopausal women aged 55-74 [13]. The results from this study demonstrated that a significant negative correlation was observed between periodontal disease and truncal bone BMD further confirming the relationship between osteoporosis and periodontal disease [13]. Further research groups studying populations from Jordan [12], India [9], and USA [11] demonstrate various relationships between periodontal disease and osteoporosis. Noteworthy, while a positive correlation is observed in some studies, other studies have failed to demonstrate either a relationship exists or have concluded that osteoporosis is not a causal factor in the development of moderate to severe chronic periodontitis $[15,16]$.

It may thus be concluded that the relationship between periodontal disease and osteoporosis is one area of research needing a more developed understanding. It is believed that osteoporosis is able to contribute to periodontal breakdown given that it may increase bone resorption and prevent proper healing ultimately increasing the severity of the preexisting periodontal disease; however, very little evidence is available demonstrating such a relationship. Therefore, the aim of the present study was to investigate with an animal model the relationship between periodontal disease progression and osteoporosis. An ovariectomized rat was chosen as an osteoporosis mod$\mathrm{el}$, and periodontal progression was induced by ligature placement of the rat mandibular first molars. Micro-CT and histological assessment were performed at $0,3,7$, and 11 days following ligature placement in shamoperated and ovariectomized (OVX) animals to assess the rate of bone loss.

\section{Materials and methods}

\section{Animal experiments}

Sixty-four female Wistar rats (7 weeks of age, 180-200 g) were used in this study. At the start of the experiment, no clinical signs of gingival inflammation were observed among the animals. The animals were housed in individual wire cages in a temperature- and humidity-controlled room $\left(23 \pm 1{ }^{\circ} \mathrm{C}\right.$ and $60 \pm 5 \%$ relative humidity) with a 12-h light/dark cycle as previously described [17]. All animals were allowed to acclimate to the laboratory environment for 1 week before surgical procedures were carried out. For 32 of the 64 animals, ovariectomy (OVX) surgery was performed at 8 weeks of age as previously described $[18,19]$. Briefly, general anesthesia was achieved by intraperitoneal injection of chloral hydrate $(10 \%, 4 \mathrm{ml} / \mathrm{kg}$ body weight); the rats were operated with a $10-\mathrm{mm}$ linear bilateral lumbar lateral skin incisions. Then, the enterocoelia was exposed by blunt dissection of muscle and peritoneum. The bilateral ovaries were removed gently following ligation of the ovarian artery and vein. Then, the small incisions were sutured stratified. Postoperatively, penicillin $(40,000 \mathrm{IU} / \mathrm{ml}$, $1 \mathrm{ml} / \mathrm{kg}$ ) was injected for 3 days, and there was no sign of inflammation or other notable anomaly. Thirty-two control animals were subject to sham operations as previously described [20].

At 8 weeks following the surgery, cotton thread ligatures (no. 3-0) were tied around the cervixes of the bilateral first mandibular molar (M1) under anesthesia with chloralic hydras for all 64 rats. At time points $0,3,7$, and 11 after ligature placement, 12 animals were euthanized (six control shamoperated animals with ligatures and six OVX animals with ligatures). The mandible was harvested and fixed in $10 \%$ formalin solution for $48 \mathrm{~h}$. After fixation, alveolar bone was examined using a micro-CT scanner and embedded in paraffin for histological analysis. 


\section{$\mu \mathrm{CT}$ scanning and analysis}

A $\mu$ CT imaging system ( $\mu$ CT50, Scanco Medical, Basersdorf, Switzerland) was used to scan the mandibular molars and alveolar bone of all animals. Scanning was performed at $70 \mathrm{kV}$ and $114 \mu \mathrm{A}$ with a thickness of $15 \mu \mathrm{m}$ per slice in medium-resolution mode and 300-ms integration time. A Gaussian filter (sigma $=0.8$ and support $=1$ ) was used to remove noise. Vertical bone loss was evaluated by measuring the distance between the cemento-enamel junction (CEJ) and the alveolar bone crest $(\mathrm{ABC})$ at six points (lingual-mesial, lingual-middle, lingual-distal, buccal-mesial, buccal-middle, buccal-distal) of the first mandibular molar. The architectures of interradicular bone of the first molar were evaluated by means of $3 \mathrm{D}$ reconstruction. An area with a height of $2.4 \mu \mathrm{m}$ was defined as the 3D regions of interest (ROIs), starting coronally by a $2 \mathrm{D}$ slice $15 \mu \mathrm{m}$ below the furcation of the first molar. Bone volume fraction was calculated from the bone volume (BV) and total volume (TV) as BV/TV. Trabecular thickness ( $\mathrm{Tb}$.Th) was calculated by applying the distance transformation by filling maximal spheres in the bone structures as determined by the micro-CT supplier protocol.

\section{Histological analysis and tartrate resistant acid phosphatase stain}

After fixation, the mandibles were decalcified in $10 \%$ EDTA, dehydrated, embedded in paraffin, sectioned to 4- $\mu \mathrm{m}$ thickness, and stained with hematoxylin and eosin (H\&E) as previously described [21]. Vertical alveolar bone loss of the first mandibular molar were assessed for inflammation and alveolar bone destruction as assessed by measuring the length $(\mathrm{mm})$ of the $\mathrm{CEJ}$ to $\mathrm{ABC}$ between the first and second mandibular molars.

Then, tartrate-resistant acid phosphatase (TRAP) (Sigma \#387A; Sigma-Aldrich, St. Louis, USA) was performed to determine osteoclast activity as previously described [21]. Analysis of the furcation area was assessed for the presence of osteoclasts as defined by cells with greater than three nuclei and counted at the various time points as previously described [22]. Samples were assessed for their staining intensity with respect to alveolar bone loss adjacent to the periodontal ligament in the furcation area along the PDL-bone perimeter.

\section{Statistical analysis}

All data analysis was performed using SPSS software and statistically significant values were adopted as $p<0.05$. All experiments were displayed as mean and standard deviation (SD) and were calculated by one-way ANOVA and Newman Keuls $t$ test.

\section{Results}

\section{Micro-CT analysis}

Micro-CT was performed to analyze the rate of alveolar bone loss in both sham-operated and OVX animals (Fig. 1, 64 total animals, six per group per time point). At day 0 , the roots embedded within alveolar bone demonstrate no differences in the vertical bone loss as assessed by micro-CT (Fig. 2). Furthermore, cross-sectional analysis from the micro-CT images reveal large areas of bone in both sham-operated (Fig. 1e) and OVX groups (Fig. 1m) with no differences in furcation bone loss between sham-operated and OVX groups (Fig. 3). At 3 days following placement of the ligature on the first mandibular molar, small noticeable changes were observed specifically on the distal root where the micro-CT images begin to demonstrate slight bone loss (Fig. 1b, i). Significantly greater vertical bone loss was observed between OVX and sham-operated animals, and this observation was maintained throughout the remaining study time points ( $p<0.01$, Fig. 2). At both 3 and 7 days, no significant differences in furcation bone loss were observed (Fig. 3). By 11 days, micro-CT images depict significantly greater bone loss in both vertical height and within the furcation in the OVX group $(p<0.01$, Figs. 11, 2, and 3) when compared to sham-operated animals (Fig. 1d). Cross-sectional analysis from micro-CT further reveals the loss of bone volume in the furcation area (Fig. 1p), and computation analysis from the micro-CT demonstrated that BV/TV as well as trabecular thickness is now significantly reduced in the furcation area in OVX animals when compared to control sham-operated animals $(p<0.01$, Fig. 3$)$.

\section{Histological analysis}

Histological analysis was performed in the furcation areas of first mandibular molars to further confirm the rate of bone loss between OVX and sham-operated animals as well as to assess osteoclast activity via TRAP staining. The histological analysis demonstrates that both sham-operated and OVX animals show healthy bone in furcation areas with little to no changes between groups (Fig. 4a, e). At 3 days postligature placement, noticeable changes in the morphology of trabecular bone became apparent with loss of bone beginning in the furcation area in both sham-operated and OVX animals demonstrating the effectiveness of the ligature-induced periodontitis model (Fig. 4b, f). At 7 days, this trend was also consistent with more bone loss taking place in the OVX group (Fig. 4c, g). By 11 days, a significantly greater amount of bone was lost in the furcation areas between OVX and sham-operated groups further confirming the micro-CT results (Fig. 3).

Then, histological quantification was used to measure the rate of vertical bone loss by calculating the distance between the $\mathrm{CEJ}$ and the $\mathrm{ABC}$ between the first and second mandibular 
Day 0

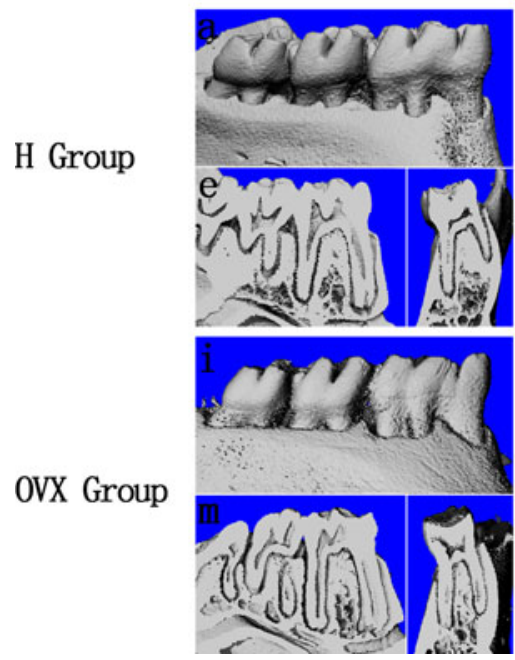

Day 3

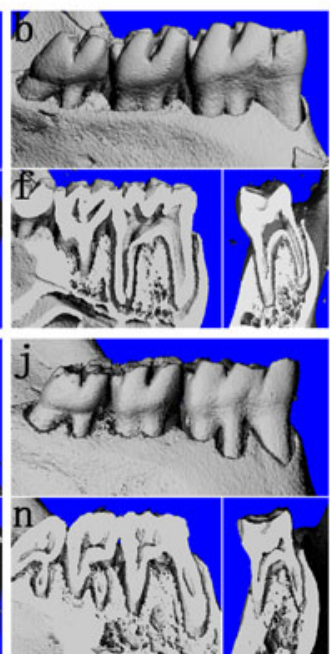

Day 7

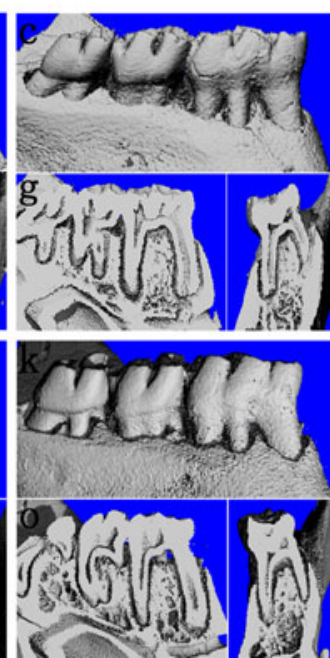

Day 11

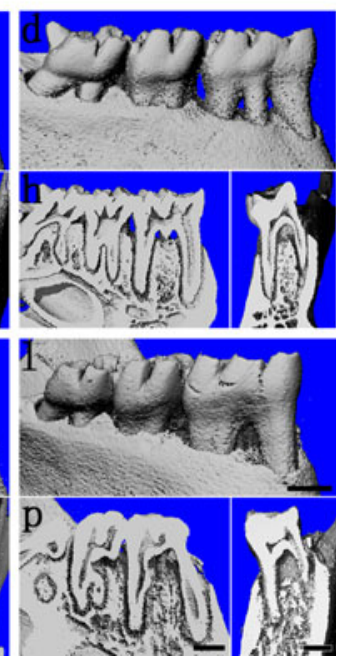

Fig. $1 \mu \mathrm{CT}$ images of alveolar bone loss in both sham-operated and OVX animals at $0,3,7$, and 11 days following ligature-induced periodontitis $($ bar $=$ $1 \mathrm{~mm})$. Lingual view $(a, b, c, d, i, j, k$, and $l)$ and cross sections $(e, f, g, h, m, n, o$, and $p$ ) of mandibles were observed for both groups

molars (Fig. 5). The histological measures further confirm micro-CT results by demonstrating a significantly greater loss of vertical bone at 3,7 , and 11 days postligature placement $(p<0.05$, Fig. 5). TRAP staining was then utilized to assess the involvement of osteoclast activity (Fig. 6). It was observed that at 3 days following ligature placement, osteoclast activity peaked with a significantly higher number of multinucleated cells and staining intensity observed in the OVX group when compared to control sham-operated samples at all time points $(p<0.01$, Fig. 6c).

\section{Discussion}

The aim of the present study was to investigate the relationship between osteoporosis and periodontal disease. There

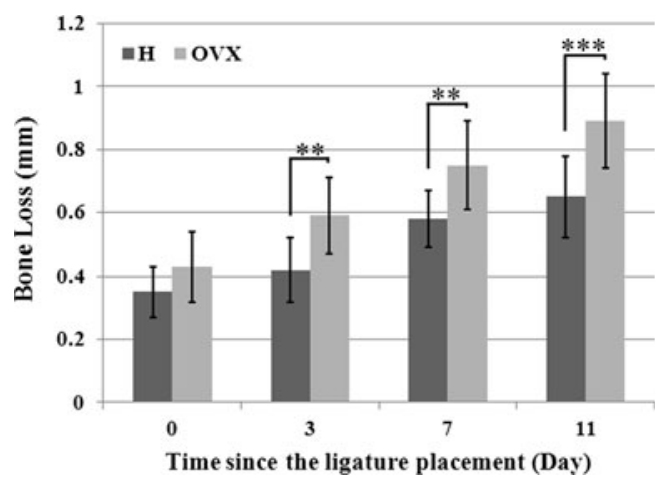

Fig. 2 Representative bar graph demonstrating vertical bone loss of the first mandibular molars as assessed by $\mu \mathrm{CT}$ images. Significantly greater vertical bone loss is observed in OVX animals at 3, 7, and 11 days postligature-induced periodontitis. Data are expressed as mean \pm standard deviation $(\mathrm{mm})\left({ }^{*} p<0.05\right)$ exists accumulating evidence that a relationship may exist [8-14]; however to date, no animal or histological study has fully confirmed this possible relationship. Therefore, we sought to utilize an animal model to perform histological assessment and to characterize alveolar bone loss in an osteoporotic model by creating OVX animals and comparing them to control sham-operated animals for periodontal changes from 0 to 11 days following a ligature-induced periodontitis animal model.

The animal model chosen was selected as it has a long history of being utilized to examine periodontal change in periodontitis [23-25]. It is noteworthy to mention that in the present study, periodontal breakdown was calculated by evaluating alveolar bone loss as the key changes associated between mucositis and periodontitis are the loss of alveoloar bone and the direct clinical attachment loss was not calculated. Instead, the distance between the CEJ and the alveolar bone crest were measured to represent this parameter. The model was confirmed as the rate of periodontal change even in shamoperated animals was significantly altered following the placement of the ligature on the first mandibular molar thus demonstrating its effectiveness as a study model. It was observed that within only 3 days, bone loss had occurred in both control sham-operated and OVX animals confirming the fast rate of bone loss in the present animal model (Fig. 2). The group containing OVX animals without a ligature-induced model was not considered as the rate of periodontal breakdown is thought to be insignificant. As the OVX animals are left for 8 weeks following removal of their ovaries with no bone loss present, including an additional group allowing an additional 10 days of induced OVX rats was not considered. At day 0 , no difference in alveolar bone loss or tissue inflammation was apparent in OVX animals when compared to 
a

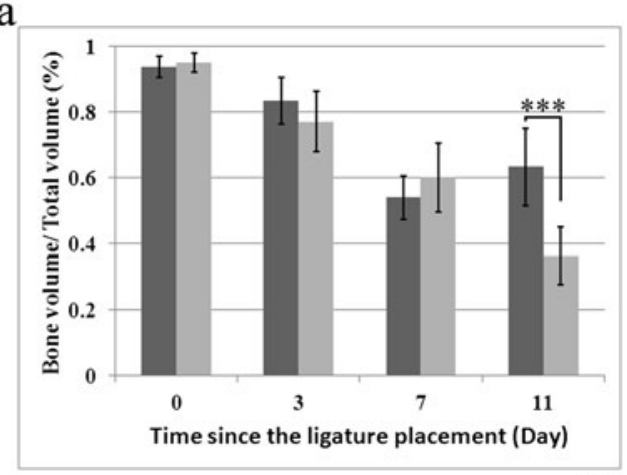

Fig. 3 a Bone volume/total volume and $\mathbf{b}$ trabecular thickness of alveolar bone located within the furcation area of the first mandibular molar. While no significant difference was observed between 0 to 7 days however at 11 days postligature-induced periodontitis, both BV/

control sham-operated animals. Including an additional 32 animals to the experimental design was thus not warranted as the rate of periodontal breakdown and alveolar bone loss is considered to be negligible. The changes occurring in the present model are apparent once the ligature-induced state was placed with more alveolar bone loss taking place in OVX animals.

The results from the present study also demonstrate the severe complication associated with the progression of both diseases. Although the present results are drawn from an animal model with a much shorter life expectancy than humans, the investigation demonstrates how quickly the degeneration of periodontal tissues may arise with a simultaneous combination of both periodontal disease and osteoporosis. It is known that periodontal disease is thought to contribute to osteoclast activation b

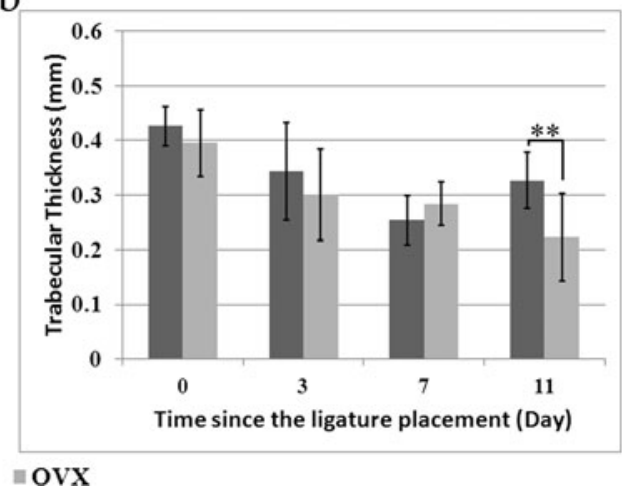

TV and trabecular thickness were significantly reduced in OVX animals when compared to control. Data are expressed as mean $\pm \mathrm{SD}(\mathrm{mm})$ $\left({ }^{*} p<0.05\right)$

[26-28] and the combination with a disease such as osteoporosis known to cause a hyper-activity of osteoclasts is subject to pose many future problems. Furthermore, it is known that both osteoporosis and periodontal disease are age-related diseases and the increasing life expectancy in humans is thought to contribute to both diseases. As patients become older, bone loss tends to increase with little therapy available to re-establish proper bone volume. Similarly, as patient age increases, their dexterity decreases making it increasingly difficult to brush their teeth efficiently and maintain proper oral hygiene. Thus, the combination of these two age-related disorders is one that necessitates full clinical attention and involvement as patients reach the later years of their lives.

We also studied the time necessary to reach the tooth furcation, an area specifically located between
Fig. 4 Representative sections of H\&E staining demonstrate periodontal breakdown of shamoperated (sham, $a-e$ ) and ovariectomized (OVX, $f-j)$ rats at $0,3,7$, and 11 days postligatureinduced peirodontitis. More alveolar bone loss is observed in the OVX group at 11 days as depicted by the loss of bone in the furcation area of first mandibular molars $(j)$. Black scale bar: $200 \mu \mathrm{m}$

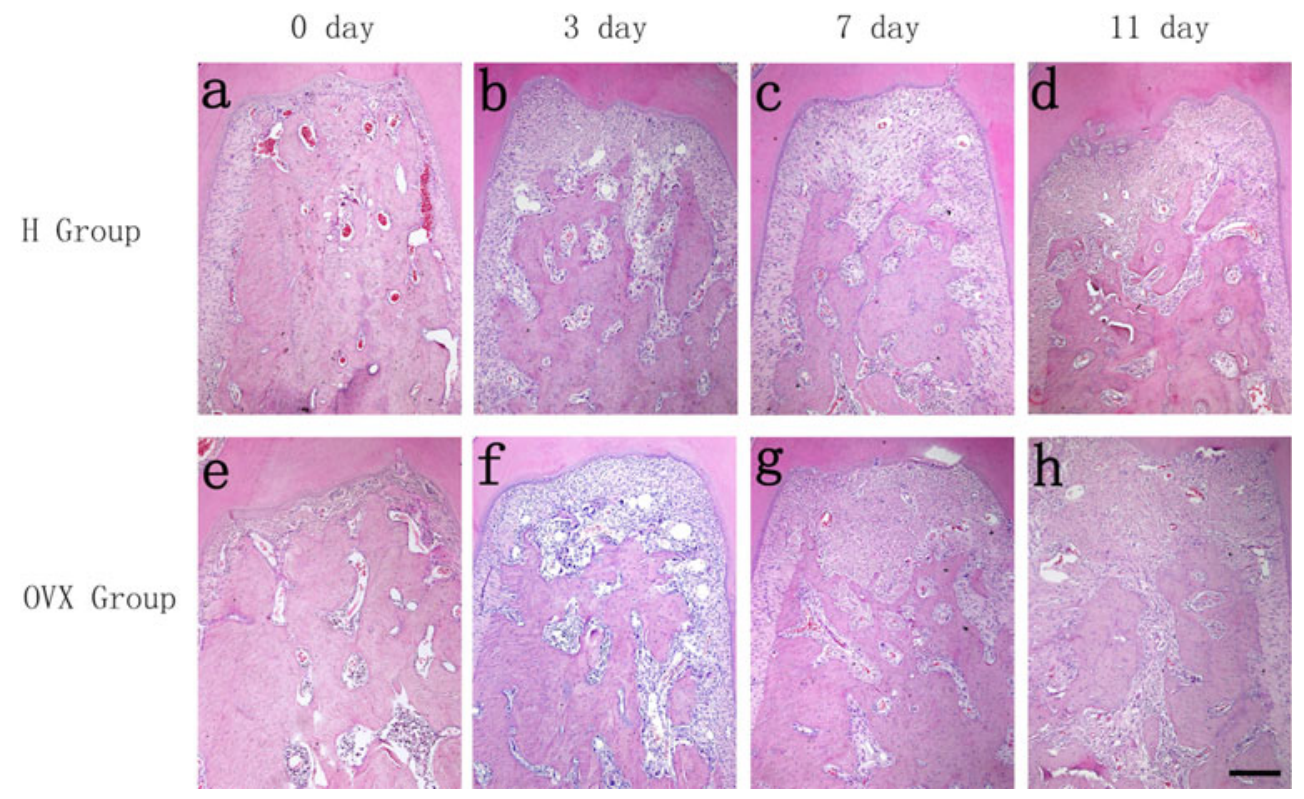




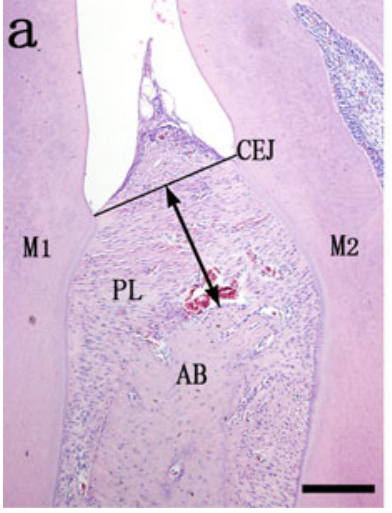

Fig. 5 The mandibular vertical alveolar bone loss in the interproximal region assessed histologically. a Histological appearance of the region between the first molar (M1) and second molar (M2) roots (bar= $200 \mu \mathrm{m}$ ). The double-headed arrow shows the vertical distance from

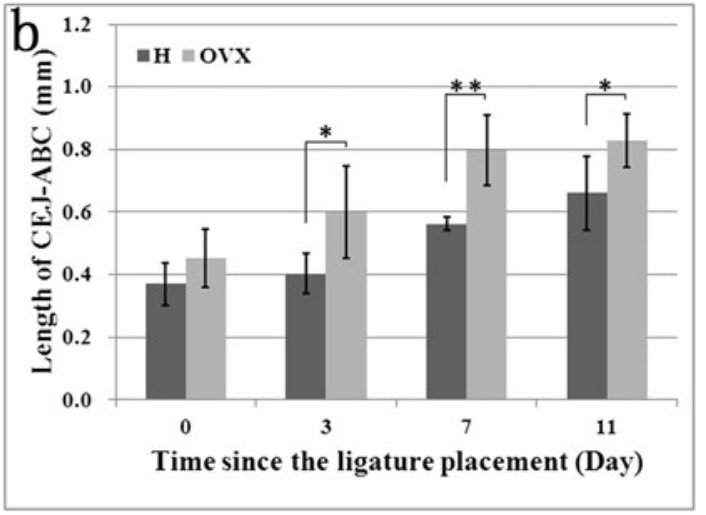

alveolar bone crest to the amelocemental junction. $C E J$ cementoenamel junction, $A B$ alveolar bone, $P L$ periodontal ligament. b Quantitative analysis of interproximal bone loss assessed in histological sections. Data are expressed as mean $\pm \mathrm{SD}(\mathrm{mm})\left({ }^{*} p<0.05, *^{*} p<0.01\right)$ the tooth roots (Fig. 5). Once bone loss exceeds the furcation area, it is known that the regenerative potential of these teeth are much more limited with no guarantee that even with the use of the best available periodontal growth factors, these teeth maintain many uncertainties involving their ability to be restored [29-31]. Thus, the results from the present study demonstrate that there is a small window from which patients can change/modify their habits which may facilitate their necessary future periodontal regeneration further illustrating the need for medical practitioners to be aware of the patient's periodontal status and act upon the diseased state in a timely manner before furcation involvement is reached.
Fig. 6 Alveolar bone resorption through TRAP $(+)$ osteoclast identification. Multinucleated osteoclast-like cells (black arrows) were observed at the PDL-bone interface for SHAM (a) and OVX (b) groups at 3 days following ligature placement ( bar $=200 \mu \mathrm{m}) . A B$ alveolar bone, $D$ dentin, $C$ cementum, $P L$ periodontal ligament. c The number of multinucleated osteoclasts within root furcation of the first molar (M1) was manually counted along the PDLbone perimeter, and ratios of osteoclast count to perimeter were calculated $\left({ }^{*} p<0.05,{ }^{* *} p<0.01\right.$, $* * * p<0.001)$
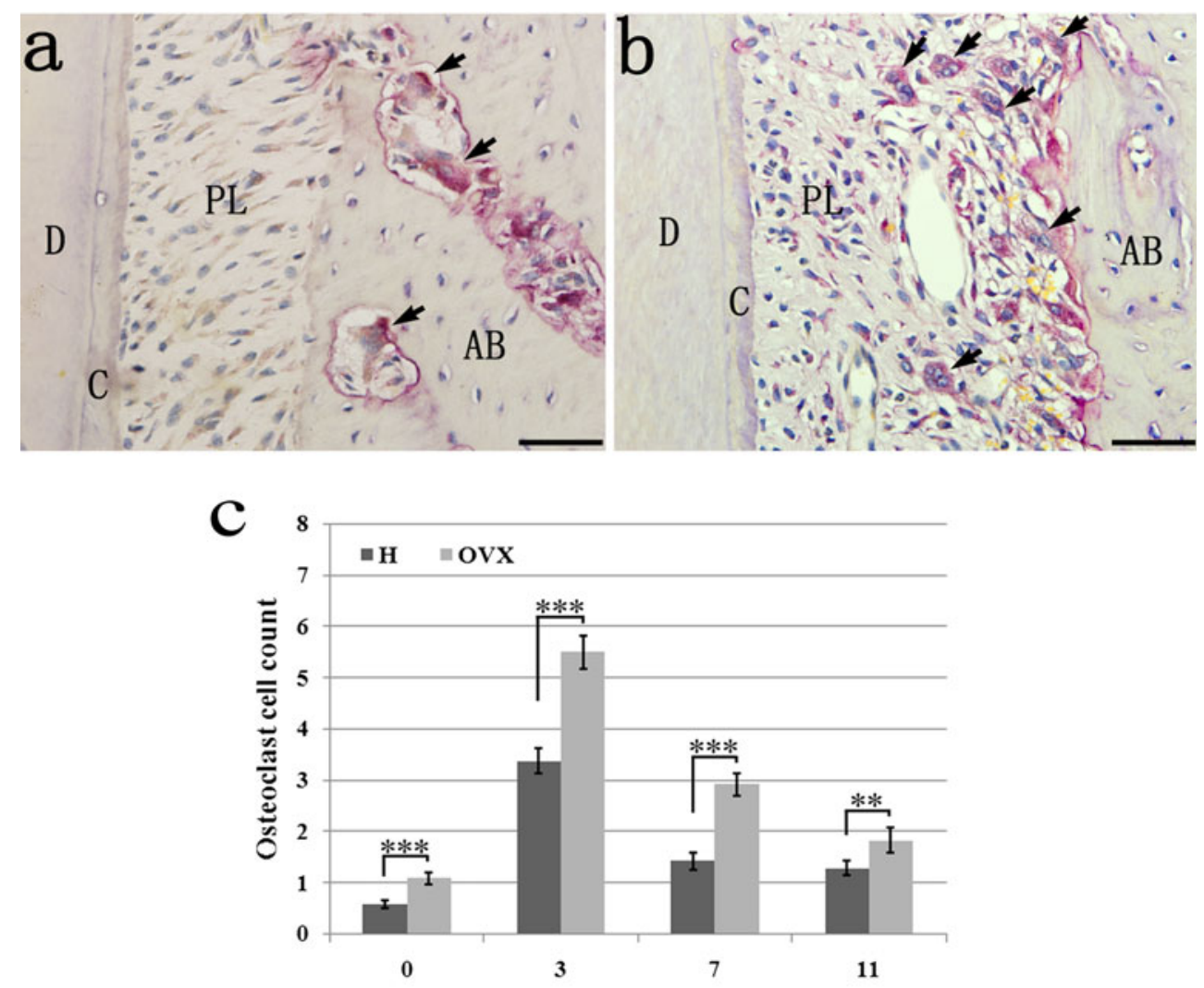

Time since the ligature placement (Day) 


\section{Conclusion}

The results from the present investigation demonstrate a correlation between osteoporosis and bone loss following ligature placement. Although osteoporosis is not thought to induce periodontal disease, we show in the present study that the disease progression was apparent in OVX animals by demonstrating significantly greater vertical bone loss, furcation bone loss, and loss of alveolar bone crest height when compared to sham-operated animals. The histological analysis reveal that OVX animals were associated with higher TRAP staining indicative that osteoclast activity was highly active in periodontal tissues when compared to their respective time point controls.

Conflict of interest Dai Jing, Yihui Ma, Miusi Shi, Zhengguo Cao, Richard J. Miron, and Yufeng Zhang declare that they have no conflict of interest. This project was supported by Program for New Century Excellent Talents in University (NCET-11-0414), Excellent Youth Foundation of Hubei, and the funds of the National Natural Science Foundation of China (81271108)

\section{References}

1. Tontonoz P, Pei LM (2004) Fat's loss is bone's gain. J Clin Invest 113:805-806. doi:10.1172/Jci200421311

2. Rodan GA, Martin TJ (2000) Therapeutic approaches to bone diseases. Science 289:1508-1514

3. Antonenko O, Bryk G, Brito G, Pellegrini G, Zeni SN (2014) Oral health in young women having a low calcium and vitamin D nutritional status. Clin Oral Investig. doi:10.1007/s00784-014-1343-x

4. Hansen PJ, Knitschke M, Draenert FG, Irle S, Neff A (2013) Incidence of bisphosphonate-related osteonecrosis of the jaws (BRONJ) in patients taking bisphosphonates for osteoporosis treatment — a grossly underestimated risk? Clin Oral Investig 17:1829_ 1837. doi:10.1007/s00784-012-0873-3

5. Marchand-Libouban H, Guillaume B, Bellaiche N, Chappard D (2013) Texture analysis of computed tomographic images in osteoporotic patients with sinus lift bone graft reconstruction. Clin Oral Investig 17:1267-1272. doi:10.1007/s00784-012-0808-z

6. Teitelbaum SL (2010) Stem cells and osteoporosis therapy. Cell Stem Cell 7:553-554. doi:10.1016/j.stem.2010.10.004

7. Silva BC, Bilezikian JP (2011) New approaches to the treatment of osteoporosis. Annu Rev Med 62:307-322. doi:10.1146/annurevmed-061709-145401

8. Passos Jde S, Gomes-Filho IS, Vianna MI, da Cruz SS, Barreto ML, Oliveira TJ, Borges LD, Monteiro FM (2010) Outcome measurements in studies on the association between osteoporosis and periodontal disease. J Periodontol 81:1773-1780. doi:10.1902/jop. 2010.100143

9. Sultan N, Rao J (2011) Association between periodontal disease and bone mineral density in postmenopausal women: a cross sectional study. Med Oral Patol Oral Cir Bucal 16:e440-e447

10. von Wowern N, Klausen B, Kollerup G (1994) Osteoporosis: a risk factor in periodontal disease. J Periodontol 65:1134-1138

11. Brennan RM, Genco RJ, Hovey KM, Trevisan M, WactawskiWende J (2007) Clinical attachment loss, systemic bone density, and subgingival calculus in postmenopausal women. J Periodontol 78:2104-2111. doi:10.1902/jop.2007.070155
12. Al Habashneh R, Alchalabi H, Khader YS, Hazza'a AM, Odat Z, Johnson GK (2010) Association between periodontal disease and osteoporosis in postmenopausal women in jordan. J Periodontol 81: 1613-1621. doi:10.1902/jop.2010.100190

13. Takahashi O, Yoshihara A, Nakamura K, Miyazaki H (2012) Association between periodontitis and systemic bone mineral density in Japanese community-dwelling postmenopausal women. J Dent 40:304-311. doi:10.1016/j.jdent.2012.01.005

14. Passos JS, Vianna MI, Gomes-Filho IS, Cruz SS, Barreto ML, Adan L, Rosing CK, Cerqueira EM, Trindade SC, Coelho JM (2013) Osteoporosis/osteopenia as an independent factor associated with periodontitis in postmenopausal women: a case-control study. Osteoporos Int J Established Result Coop Eur Found Osteoporos Natl Osteoporos Found USA 24:1275-1283. doi:10.1007/s00198012-2130-7

15. Marjanovic EJ, Southern HN, Coates P, Adams JE, Walsh T, Horner K, Devlin H (2013) Do patients with osteoporosis have an increased prevalence of periodontal disease? A cross-sectional study. Osteoporos Int J Established Result Coop Eur Found Osteoporos Natl Osteoporos Found USA 24:1973-1979. doi:10.1007/s00198012-2246-9

16. Darcey J, Devlin H, Lai D, Walsh T, Southern H, Marjanovic E, Horner K (2013) An observational study to assess the association between osteoporosis and periodontal disease. Br Dent J 215:617621. doi:10.1038/sj.bdj.2013.1191

17. Miron RJ, Wei L, Bosshardt DD, Buser D, Sculean A, Zhang Y (2014) Effects of enamel matrix proteins in combination with a bovine-derived natural bone mineral for the repair of bone defects. Clin Oral Investig 18:471-478. doi:10.1007/s00784-013-0992-5

18. Miron RJ, Wei L, Yang S, Caluseru OM, Sculean A, Zhang Y (2014) Effect of an enamel matrix derivative on periodontal wound healing/regeneration in an osteoporotic model. J Periodontol 11: 1603-1611. doi:10.1902/jop.2014.130745

19. Wei L, Ke J, Prasadam I, Miron RJ, Lin S, Xiao Y, Chang J, Wu C, Zhang Y (2014) A comparative study of Sr-incorporated mesoporous bioactive glass scaffolds for regeneration of osteopenic bone defects. Osteoporos Int J Established Result Coop Eur Found Osteoporos Natl Osteoporos Found USA 25:2089-2096. doi:10. 1007/s00198-014-2735-0

20. Zhang Y, Cheng N, Miron R, Shi B, Cheng X (2012) Delivery of PDGF-B and BMP-7 by mesoporous bioglass/silk fibrin scaffolds for the repair of osteoporotic defects. Biomaterials 33:6698-6708. doi:10.1016/j.biomaterials.2012.06.021

21. Yang S, Lan L, Miron RJ, Wei L, Zhang M, Zhang Y (2014) Variability in particle degradation of four commonly employed dental bone grafts. Clin Implant Dent Relat Res. doi:10.1111/cid. 12196

22. Zhang Y, Wei L, Miron RJ, Shi B, Bian Z (2015) Anabolic bone formation via a site-specific bone-targeting delivery system by interfering with semaphorin 4D expression. J Bone Mineral Res Off J Am Soc Bone Mineral Res 30:286-296. doi:10.1002/jbmr.2322

23. Gyorfi A, Fazekas A, Suba Z, Ender F, Rosivall L (1994) Neurogenic component in ligature-induced periodontitis in the rat. J Clin Periodontol 21:601-605

24. Nociti FH Jr, Nogueira-Filho GR, Primo MT, Machado MA, Tramontina VA, Barros SP, Sallum EA (2000) The influence of nicotine on the bone loss rate in ligature-induced periodontitis. A histometric study in rats. J Periodontol 71:1460-1464. doi:10.1902/ jop.2000.71.9.1460

25. Samejima Y, Ebisu S, Okada H (1990) Effect of infection with Eikenella corrodens on the progression of ligature-induced periodontitis in rats. J Periodontal Res 25:308-315

26. Jarry CR, Duarte PM, Freitas FF, de Macedo CG, ClementeNapimoga JT, Saba-Chujfi E, Passador-Santos F, de Araujo VC, Napimoga MH (2013) Secreted osteoclastogenic factor of activated $\mathrm{T}$ cells (SOFAT), a novel osteoclast activator, in chronic 
periodontitis. Hum Immunol 74:861-866. doi:10.1016/j.humimm. 2013.04.013

27. Niwa T, Mizukoshi K, Azuma Y, Kashimata M, Shibutani T (2013) Fundamental study of osteoclast chemotaxis toward chemoattractants expressed in periodontitis. J Periodontal Res 48: 773-780. doi:10.1111/jre.12068

28. Vernal R, Diaz-Zuniga J, Melgar-Rodriguez S, Pujol M, DiazGuerra E, Silva A, Sanz M, Garcia-Sanz JA (2014) Activation of RANKL-induced osteoclasts and memory $\mathrm{T}$ lymphocytes by Porphyromonas gingivalis is serotype dependant. J Clin Periodontol 41:451-459. doi:10.1111/jcpe. 12236

29. Casarin RC, Ribeiro Edel P, Nociti FH Jr, Sallum AW, Ambrosano GM, Sallum EA, Casati MZ (2010) Enamel matrix derivative proteins for the treatment of proximal class II furcation involvements: a prospective 24-month randomized clinical trial. J Clin Periodontol 37:1100-1109. doi:10.1111/j.1600-051X. 2010.01614.x

30. Casarin RC, Ribeiro Edel P, Ribeiro FV, Nociti FH Jr, Sallum AW, Sallum EA, Casati MZ (2009) Influence of anatomic features on the effectiveness of enamel matrix derivative proteins in the treatment of proximal Class II furcation involvements. Quintessence Int 40: 753-761

31. Gkranias ND, Graziani F, Sculean A, Donos N (2012) Wound healing following regenerative procedures in furcation degree III defects: histomorphometric outcomes. Clin Oral Investig 16:239249. doi:10.1007/s00784-010-0478-7 